\section{What makes efficient circularly polarised luminescence in the condensed phase: aggregation-induced circular dichroism and light emission $\dagger$}

\author{
Jianzhao Liu, ${ }^{a b c}$ Huimin Su, ${ }^{d}$ Luming Meng, ${ }^{a}$ Yihua Zhao, ${ }^{a}$ Chunmei Deng, ${ }^{a}$ Jason C. Y. Ng, ${ }^{a b c}$ Ping Lu, ${ }^{a}$ \\ Mahtab Faisal, ${ }^{a}$ Jacky W. Y. Lam, ${ }^{a b c}$ Xuhui Huang, ${ }^{a}$ Hongkai Wu, ${ }^{a}$ Kam Sing Wong *d \\ and Ben Zhong Tang*abce
}

\section{Received 28th March 2012, Accepted 6th June 2012}

DOI: $10.1039 / \mathrm{c} 2 \mathrm{sc20382k}$

In this contribution, we conceptually present a new avenue to construction of molecular functional materials with high performance of circularly polarised luminescence (CPL) in the condensed phase. A molecule (1) containing luminogenic silole and chiral sugar moieties was synthesized and thoroughly characterized. In a solution of 1, no circular dichroism (CD) and fluorescence emission are observed, but upon molecular aggregation, both the $\mathrm{CD}$ and fluorescence are simultaneously turned on, showing aggregation-induced CD (AICD) and emission (AIE) effects. The AICD effect is supported by the fact that the molecules readily assemble into right-handed helical nanoribbons and superhelical ropes when aggregated. The AIE effect boosts the fluorescence quantum efficiency $\left(\Phi_{\mathrm{F}}\right)$ by 136 fold $\left(\Phi_{\mathrm{F}}, \sim 0.6 \%\right.$ in the solution versus $\sim 81.3 \%$ in the solid state), which surmounts the serious limitations of aggregationcaused quenching effect encountered by conventional luminescent materials. Time-resolved fluorescence study and theoretical calculation from first principles conclude that restriction of the lowfrequency intramolecular motions is responsible for the AIE effect. The helical assemblies of $\mathbf{1}$ prefer to emit right-handed circularly polarised light and display large CPL dissymmetry factors $\left(g_{\text {em }}\right)$, whose absolute values are in the range of $0.08-0.32$ and are two orders of magnitude higher than those of commonly reported organic materials. We demonstrate for the first time the use of a Teflon-based microfluidic technique for fabrication of the fluorescent pattern. This shows the highest $g_{\text {em }}$ of -0.32 possibly due to the enhanced assembling order in the confined microchannel environment. The CPL performance was preserved after more than half year storage under ambient conditions, revealing the excellent spectral stability. Computational simulation was performed to interpret how the molecules in the aggregates interact with each other at the molecular level. Our designed molecule represents the desired molecular functional material for generating efficient CPL in the solid state, and the current study shows the best results among the reported organic conjugated molecular systems in terms of emission efficiency, dissymmetry factor, and spectral stability.

\section{Introduction}

Circularly polarised luminescence (CPL) reflects the chirality of materials in the excited electronic state. The stereochemical, conformational, and three-dimensional structure information of chiral materials can be identified from their spectroscopic CPL signatures. ${ }^{1,2} \mathrm{CPL}$ is characterized by differential spontaneous emission of left- and right-handed circularly polarised (LCP and $\mathrm{RCP})$ light upon photo- or electro-excitation $\left[\Delta I(\lambda)=I_{\mathrm{L}}(\lambda)-\right.$
${ }^{a}$ Department of Chemistry, Department of Biomedical Engineering, Institute for Advanced Study, Institute of Molecular Functional Materials, The Hong Kong University of Science \& Technology (HKUST), Clear Water Bay, Kowloon, Hong Kong, China. E-mail: tangbenz@ust.hk

${ }^{b}$ State Key Laboratory of Molecular Neuroscience, HKUST, Clear Water Bay, Kowloon, Hong Kong, China

${ }^{c}$ HKUST Fok Ying Tung Research Institute, Nansha, Guangzhou, China ${ }^{d}$ Department of Physics, HKUST, Clear Water Bay, Kowloon, Hong Kong, China.E-mail: phkswong@ust.hk

${ }^{e}$ Department of Polymer Science \& Engineering, Zhejiang University, Hangzhou 310027, China $\dagger$ Electronic supplementary information (ESI) available: Crystal data and structure refinement for 6 (Table S1), fluorescence decay parameters of 1 in a dilute solution and cast thin film (Table S2), selected reorganization energies, corresponding Huang-Rhys factors, and vibrational frequencies of the first excited state of TPS (Table S3), TGA thermogram of $\mathbf{1}$ (Fig. S1), absorption spectra of $\mathbf{1}$ in DCM/hexane mixtures and dispersed in PMMA matrix. (Fig. S2), electron diffraction pattern of aggregates of $\mathbf{1}$ in DCM/hexane mixture (Fig. S3), calculated energies of different packing modes of 1 (Table S4), and structural analysis of the intermolecular interactions (Fig. S4). CCDC reference number 845739. For ESI and crystallographic data in CIF or other electronic format see DOI: $10.1039 / \mathrm{c} 2 \mathrm{sc} 20382 \mathrm{k}$ 
$I_{\mathrm{R}}(\lambda)$, where $I_{\mathrm{L}}(\lambda)$ and $I_{\mathrm{R}}(\lambda)$ denote the emission intensities of LCP and RCP component, respectively]. ${ }^{1}$ Chiral materials with efficient CPL are very useful for bio-sensing and optoelectronic applications, such as probing biomacromolecular targeting/ binding events by reading the change of CPL signal, and devices for stereoscopic optical information processing, display and storage. ${ }^{3-6}$ All these technological applications require materials with high CPL performance. Two essential parameters are utilized to evaluate the performance of CPL-active materials: one is the emission dissymmetry factor $g_{\mathrm{em}}$, which is defined as $2\left(I_{\mathrm{L}}-\right.$ $\left.I_{\mathrm{R}}\right) /\left(I_{\mathrm{L}}+I_{\mathrm{R}}\right)$ and whose value is in the range of -2 to 2 , and the other is the emission efficiency, especially in condensed phase for practical use. So far, various chiral systems, such as lanthanide ion complexes, ${ }^{7,8}$ transition metal complexes, ${ }^{9-11}$ organic molecules, ${ }^{12-15}$ synthetic polymers, ${ }^{16-24}$ biomacromolecules, ${ }^{3}$ and crystals, ${ }^{13}$ have been reported with CPL observations. However, most of them were studied in solution phase and were found to have small $g_{\mathrm{em}}$ values $\left(\sim 10^{-5}-10^{-2}\right)$, except for some rare earth compounds $^{7,8}$ and liquid crystalline conjugated polymers., ${ }^{4,20}$ From solution to condensed phase, the performance becomes even worse because aggregation of chiral luminophores normally populates the nonradiative pathways and thus quenches the light emission to a great extent. In this context, development of new chiral luminescent systems with both high emission efficiency and a large dissymmetry factor in the condensed phase has been a challenging task.

Organic chiral $\pi$-conjugated molecules are some of the most promising candidate materials for uses in advanced electronic CPL devices due to their tailored synthetic feasibility, low cost, and high flexibility and processability. In principle, helically organized luminophores can produce a CPL signal upon photoor electro-excitation. ${ }^{1}$ A common strategy is to synthesize molecules with chiral moieties at the peripheries and planar $\pi$ conjugated luminophores in the core as shown in Fig. 1a and helical assembly can be formed under the driving force of $\pi-\pi$ stacking interactions. Due to the formation of detrimental excimers or exciplexes induced by $\pi-\pi$ stacking interactions in the aggregate state, the CPL of the assembly suffers from intrinsic aggregation-caused quenching (ACQ) effect, ${ }^{25}$ leading to poor emission efficiency and spectral instability. From the practical application point of view, high emission efficiency and spectral stability are exceptionally necessary.

To overcome the serious limitation of ACQ, efficient solid emitters by judicious construction strategy are needed. An exactly opposite phenomenon to the ACQ effect was observed by Tang's group. ${ }^{26} \mathrm{~A}$ family of propeller-shaped $\pi$-conjugated molecules, for example, silole and tetraphenylethene, which are weakly/non-emissive in dilute solutions, are induced to emit intensively by aggregate formation, with the fluorescence quantum efficiency $\left(\Phi_{\mathrm{F}}\right)$ enhanced by more than two orders of magnitude. This novel phenomenon was termed as "aggregationinduced emission" (AIE). ${ }^{26-28}$ With this inspiration, we conceive an idea that an appropriate welding of AIE-active luminophore and chiral moieties may open up a new avenue to creating high performance CPL-active materials, which ideally meet the requirements of high emission efficiency and large dissymmetry factor in the aggregate state (Fig. 1b). As a proof of concept, in this contribution, we designed molecule $\mathbf{1}$ containing an AIEactive silole core and chiral sugar pendants as shown in
Scheme 1. This molecule has no circular dichroism (CD) and is nonemissive in solution. Upon aggregation, 1 can easily assemble into hierarchical helical structures and displays simultaneous aggregation-induced CD (AICD) and AIE characteristics, giving absolute $\Phi_{\mathrm{F}}$ and $g_{\mathrm{em}}$ values up to $81.3 \%$ and $\sim 0.32$ in the solid state, respectively. We present experimental and theoretical evidence to demonstrate how our new construction strategy works. To the best of our knowledge, this system represents the best result among organic chiral $\pi$-conjugated molecule systems in terms of emission efficiency, dissymmetry factor, and spectral stability.

\section{Results and discussion}

\section{Synthesis, structural characterisation, and thermal stability}

With a view to making molecules with high CPL performance in the condensed phase, which requires both high $\mathrm{CD}$ activity and emission efficiency in the aggregate state, we came up with a strategy as shown in Fig. 1b by integrating AIE luminophore with chiral blocks. We selected silole as the AIE luminophore and mannose as the chiral component, and covalently connected them via an azide-alkyne click route (Scheme 1). ${ }^{29}$ Diazidefunctionlized silole $\mathbf{6}$ was the key reactant and was prepared by using published experimental procedures with some modifications. ${ }^{30,31}$ In the presence of lithium naphthalenide (LiNaph), dimethylbis(phenylethynyl)silane (2) undergoes intramolecular reductive cyclization in an endo-endo mode to form 2,5-dilithiosilole (3), which is transformed to 2,5-dizinc silole (4) by the transmetalation with $\mathrm{ZnCl}_{2} \cdot$ TMEDA (TMEDA $=\mathrm{N}, \mathrm{N}, \mathrm{N}^{\prime}, \mathrm{N}^{\prime}-$ tetramethylethylenediamine). The subsequent reaction of $\mathbf{4}$ with 4-bromobenzylazide (5) in the presence of a palladium catalyst yields the desired product 1,1-dimethyl-2,5-bis[4-(azidomethyl) phenyl]-3,4-diphenylsilole (6). The structure of $\mathbf{6}$ has been confirmed by single crystal X-ray diffraction analysis (Scheme 1 and Table S1 $\uparrow$ ). Click reactions of two equivalents of $7^{32}$ with 6 were catalyzed by $\mathrm{CuSO}_{4} /$ sodium ascorbate, furnishing $\mathbf{1}$ in $\sim 96 \%$ yield. The titled compound $\mathbf{1}$ was characterized by standard spectroscopic techniques including ${ }^{1} \mathrm{H}$ NMR, ${ }^{13} \mathrm{C}$ NMR, and HRMS, from which satisfactory analysis data corresponding to their expected chemical structures were obtained (see Experimental Section for detailed spectroscopic data). The thermal stability of $\mathbf{1}$ was evaluated by thermogravimetric analysis (TGA). It loses $5 \%$ of its original weight when it is heated to a temperature of about $314{ }^{\circ} \mathrm{C}\left(T_{\mathrm{d}}\right)$ (Fig. $\left.\mathrm{S} 1 \dagger\right)$, suggestive of its high resistance to thermolytic attacks.

\section{Electronic transition and circular dichroism}

The molecule 1 has a specific optical rotation $\left([\alpha]_{\mathrm{D}}^{23}\right)$ of $+24.6^{\circ}$ $\left(c=0.26 \mathrm{~g} \mathrm{dL}^{-1}\right.$ in DCM). In DCM solution, 1 shows two absorption bands located at 360 and $279 \mathrm{~nm}$, corresponding to the absorptions of the silole core and the peripheral triazolylphenyl rings, respectively (Fig. 2a). Addition of hexane, a nonsolvent of $\mathbf{1}$, to the DCM solution can cluster the molecules into aggregates and form a suspension. The absorption spectra of 1 in DCM/hexane mixtures are shifted a little compared to that in pure DCM and keep almost unchanged with the concentrations (Fig. S2 $\dagger$ ). CD spectroscopy, which measures the differential absorption of LCP and RCP light, $\left[\Delta \varepsilon(\lambda)=\varepsilon_{\mathrm{L}}(\lambda)-\varepsilon_{\mathrm{R}}(\lambda)\right.$, where 


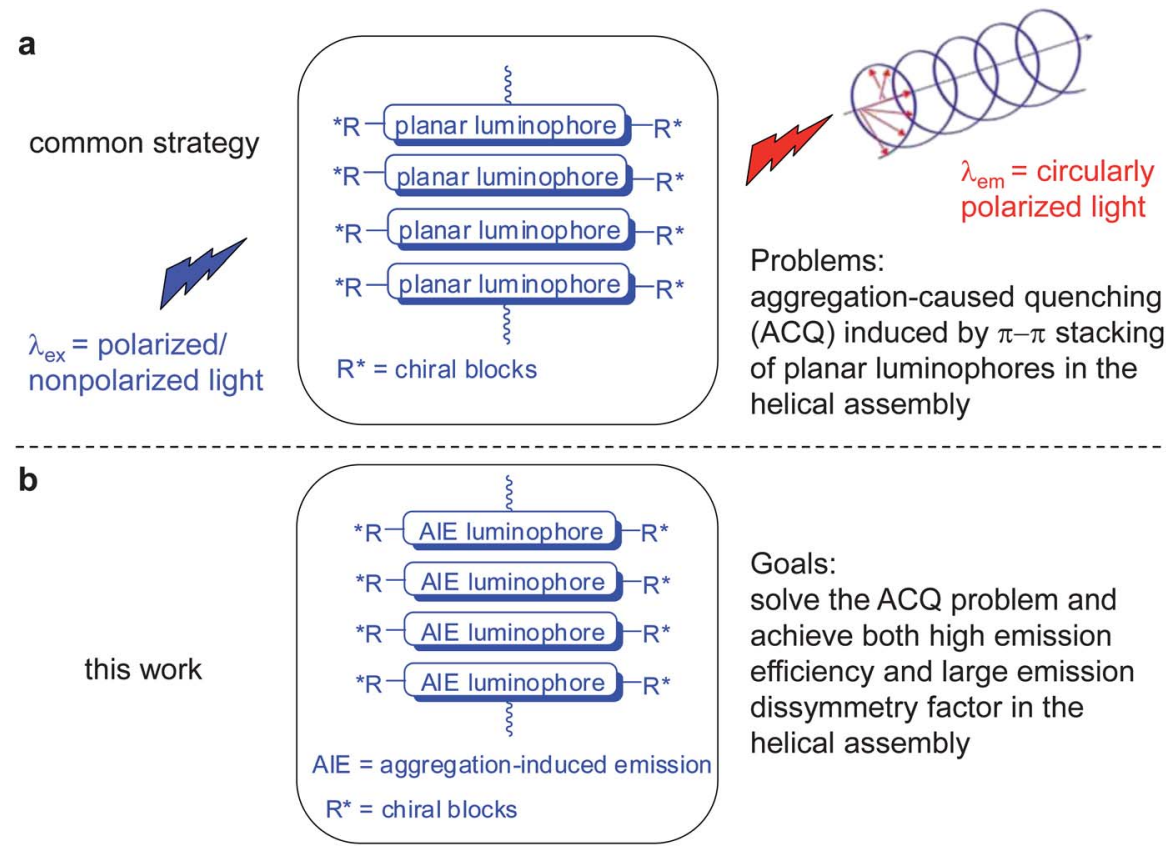

Fig. 1 Comparison of molecular design strategies for making CPL-active materials. CPL (output $\lambda_{\mathrm{em}}$ ) is generated from helical assemblies of luminescent chiral molecules bearing (a) conventional planar and (b) AIE luminophores under the excitation of polarised/nonpolarised light (input $\lambda_{\text {ex }}$ ).

$\varepsilon_{\mathrm{L}}(\lambda)$ and $\varepsilon_{\mathrm{R}}(\lambda)$ are the molar extinction coefficients of LCP and RCP light, respectively], mirrors the structural information of the ground electronic state of a system. ${ }^{33}$ Fig. $2 b$ shows the CD spectra of 1 in DCM solutions with different concentrations and all of them are CD-silent in the absorption region. However, in $\mathrm{DCM} /$ hexane mixture $(1 / 9 \mathrm{v} / \mathrm{v})$ with a concentration of $10^{-5} \mathrm{M}$, aggregation of 1 leads to the emergence of Cotton effects at 278 and $249 \mathrm{~nm}$, which are attributed to the absorptions of triazolylphenyl group (Fig. 2c), showing an AICD effect. With the concentration increased from $1 \times 10^{-5}$ to $2 \times 10^{-4} \mathrm{M}$, a new peak at $\sim 340 \mathrm{~nm}$ appears and grows stronger with respect to the peak at $278 \mathrm{~nm}$. This new peak is considered to be associated with the absorption transition of the silole core, suggesting the occurrence of chirality transfer that the chiral sugar pendant together with triazolylphenyl group induces the silole core to be helically arranged with a preferred screw sense. From $2 \times 10^{-5}$ to

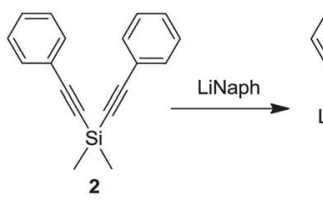

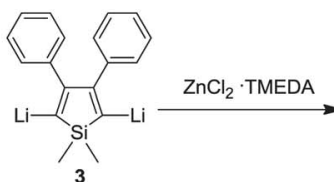


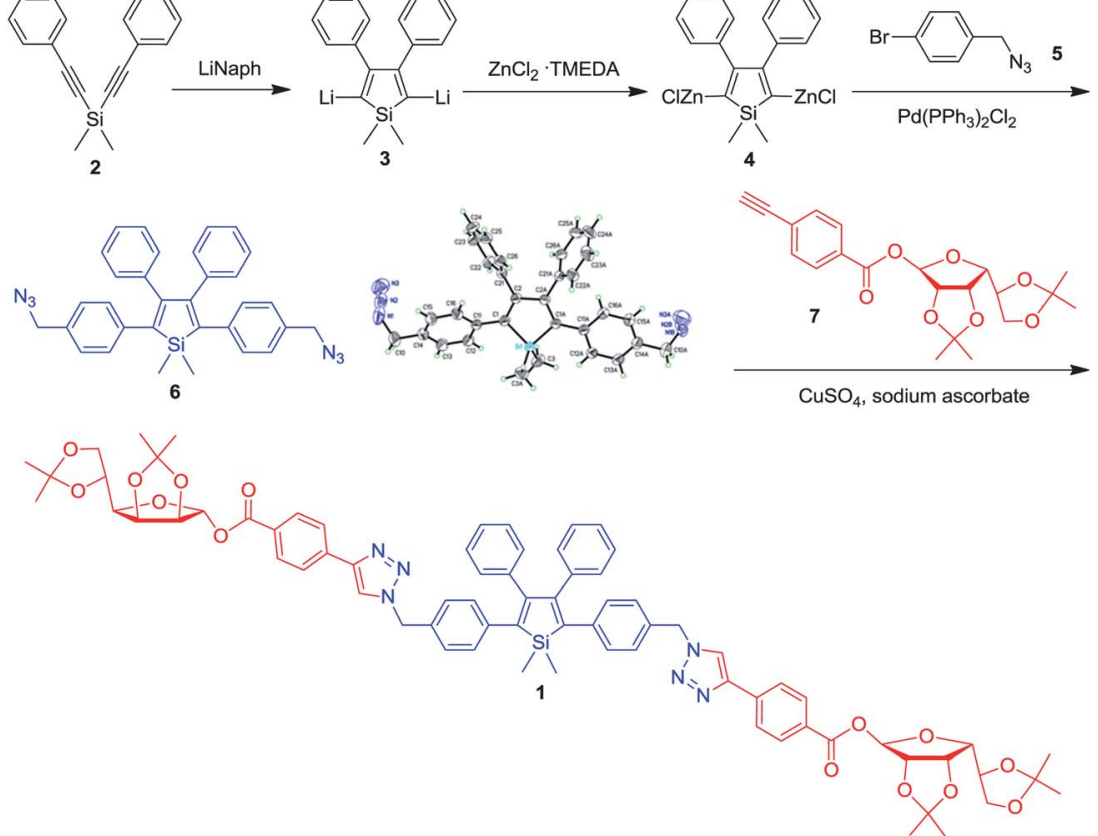

Scheme 1 Synthetic route to chiral AIE-active molecule 1 with CPL activity according to the strategy shown in Fig. 1b. The ORTEP drawing of single crystal structure of intermediate 6 at $40 \%$ probability is shown. 

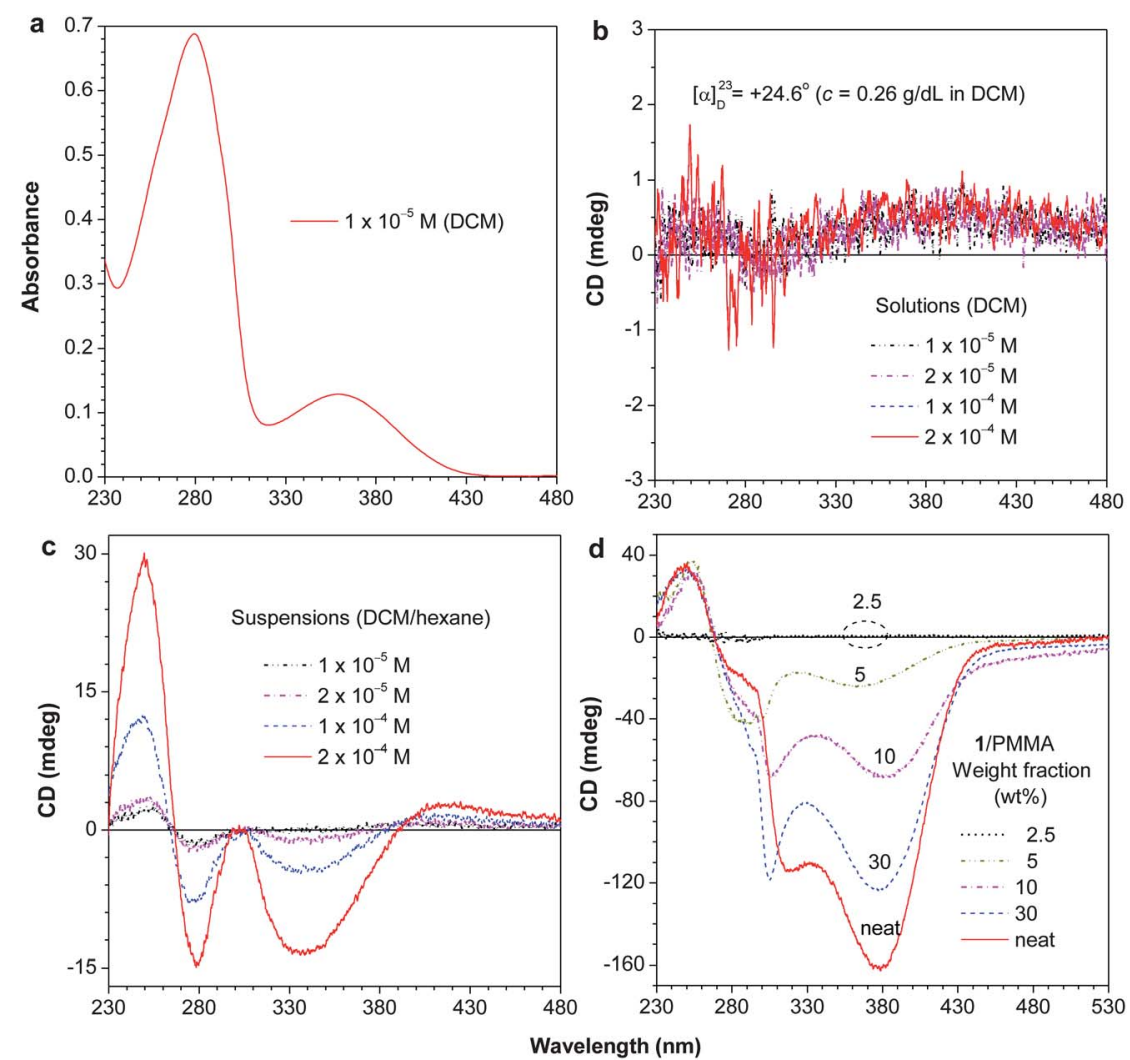

Fig. 2 Properties of electronic transition and circular dichroism. (a) Absorption spectrum of $\mathbf{1}$ in DCM. (b and $\mathbf{c}$ ) CD spectra of $\mathbf{1}$ with different concentrations in (b) DCM solutions and (c) DCM/hexane (v/v 1/9) mixtures, respectively. (d) CD spectra of 1 with different weight fractions (wt\%) dispersed in PMMA matrix prepared by drop casting of their mixed DCE solutions. Concentration of PMMA $=10 \mathrm{mg} \mathrm{mL}^{-1}$. The CD spectrum of neat 1 prepared by natural evaporation of its DCE solution of $2 \mathrm{mg} \mathrm{mL}^{-1}$ on a quartz substrate is also shown for comparison.

$2 \times 10^{-4} \mathrm{M}$, the absorption dissymmetry factor at $360 \mathrm{~nm}, g_{\text {abs }}=$ $2\left[\varepsilon_{\mathrm{L}}(\lambda)-\varepsilon_{\mathrm{R}}(\lambda)\right] /\left[\varepsilon_{\mathrm{L}}(\lambda)+\varepsilon_{\mathrm{R}}(\lambda)\right],{ }^{33}$ is increased from 1.59 to $2.23 \times$ $10^{-3}$. Fig. $2 \mathrm{~d}$ reveals the CD spectra of cast films of $\mathbf{1}$ dispersed in poly(methyl methacrylate) (PMMA) matrix with different weight fractions $(\mathrm{wt} \%)$. Below $2.5 \mathrm{wt} \%$, no CD signal is detected. When more than $5 \mathrm{wt} \%$ of $\mathbf{1}$ is loaded, there is a trend that the intensity of $\mathrm{CD}$ signal increases with the loading amount. It is understandable that at a small loading ratio, $\mathbf{1}$ exists in an isolated state in the polymer matrix, whereas with a larger amount loaded, the molecules tend to aggregate due to the intrinsic phase separation effect and the population of chiral aggregates with helical structures becomes larger accordingly. In addition, the two AICD peaks originally at 340 and $278 \mathrm{~nm}$ in DCM/hexane suspensions are red-shifted to $\sim 380$ and $\sim 300 \mathrm{~nm}$, respectively, and the former peak becomes more intense, indicative of the formation of a more conjugated structure in the helical assemblies and thus the enhanced chirality transfer effect. Natural evaporation of neat $\mathbf{1}$ in 1,2-dichloroethane (DCE) solution on a quartz substrate renders the formation of non-smooth film, which shows a similar spectral profile to those mentioned above. The CD red-shift is consistent with the absorption behaviour (Fig. S2†).

\section{Aggregation-induced emission (AIE)}

As anticipated, the designed molecule $\mathbf{1}$ is AIE-active: it has faint photoluminescence (PL) when molecularly dissolved in its good solvents but is induced to fluoresce intensively when aggregated in poor solvents (Fig. 3). The fluorescence intensity and $\Phi_{\mathrm{F}}$ value of $\mathbf{1}$ is almost unchanged until $\sim 80 \mathrm{vol} \%$ poor solvents are added. Afterwards, the fluorescence efficiency rises abruptly. In pure solutions, the $\Phi_{\mathrm{F}}$ value of $\mathbf{1}$ is merely $0.6 \%$, whereas in the DCM/ hexane mixtures with $90 \mathrm{vol} \%$ hexane, its $\Phi_{\mathrm{F}}$ value rises to $31.5 \%$ as clearly discerned by the fluorescence images shown in Fig. $3 \mathrm{~b}$. It is amazing that the $\Phi_{\mathrm{F}}$ value in solid thin film state can reach $81.3 \%$. Meanwhile, the profiles of the PL spectra keep unchanged from solution to aggregate state. The AIE effect can be quantified by the extent of emission enhancement $\left(\alpha_{\mathrm{AIE}}\right)$, as defined by the following equation

$$
\alpha_{\mathrm{AIE}}=\frac{\Phi_{\mathrm{F}(\mathrm{aggr})}}{\Phi_{\mathrm{F}(\mathrm{soln})}}
$$

where $\Phi_{\mathrm{F}(\text { aggr })}$ and $\Phi_{\mathrm{F}(\text { soln })}$ are the quantum efficiencies in the aggregate (e.g., the $\Phi_{\mathrm{F}}$ value in the thin film state) and solution states, respectively. According to eqn (1), the corresponding $\alpha_{\mathrm{AIE}}$ factor of $\mathbf{1}$ is about 136.

\section{Fluorescence decay dynamics and theoretical understanding from first principles}

To deepen our understanding of the AIE phenomenon of 1, we studied its emission dynamic by time-resolved technique. Lifetime is a key kinetic parameter for the PL decay. In dilute solution, the excited state of $\mathbf{1}$ decays in a single-exponential fashion, 



Fig. 3 Photoluminescent properties and theoretical calculations. (a) PL spectra of 1 in DCM/hexane mixtures with different volume fractions $\left(f_{\mathrm{H}}\right)$ of hexane. Concentration $=10^{-5} \mathrm{M}$; excitation wavelength $=356 \mathrm{~nm}$. (b) Fluorescence quantum yield $\left(\Phi_{\mathrm{F}}\right)$ of 1 versus solvent composition of the DCM/ hexane mixture. The $\Phi_{\mathrm{F}}$ 's were estimated using 9,10 -diphenylanthrance as standard ( $\Phi_{\mathrm{F}}=90 \%$ in cyclohexane). The $\Phi_{\mathrm{F}}$ value of thin cast film ( $\left.\Phi_{\mathrm{F}, \mathrm{f}}\right)$ of 1 determined by integrating sphere is given in panel $\mathbf{b}$ for comparison. Inset of panel $\mathbf{b}$ : fluorescent photographs of $\mathbf{1}$ in DCM/hexane mixtures with $f_{\mathrm{H}}$ 's of 0 and 90 and its powder under a hand-held UV lamp with an excitation wavelength of $365 \mathrm{~nm}$. (c) Time-resolved fluorescence decay curves of 1 in DCM solution with a concentration of $10^{-5} \mathrm{M}$ and its cast thin film from a DCE solution of $2 \mathrm{mg} \mathrm{mL}^{-1}$. (d) Plot of calculated reorganization energy versus the normal mode wavenumber of TPS.

suggesting that all the excited molecules relax through the same pathway (Fig. 3c). The lifetime is as short as $\sim 30$ ps (Table S2†). In the solid thin film state, the PL decay is much slower and the decay dynamics are better fitted by a double-exponential function. This indicates that two relaxation pathways are involved in the decay process. For example, $39 \%\left(A_{1}\right)$ and $61 \%\left(A_{2}\right)$ of the excitons of $\mathbf{1}$ decay via the fast and slow channels with lifetimes of $0.46 \mathrm{~ns}\left(\tau_{1}\right)$ and $2.49 \mathrm{~ns}\left(\tau_{2}\right)$, respectively. The weighted mean lifetime $(\tau)$ is $1.70 \mathrm{~ns}$. These results are consistent with the steady state PL studies. In addition, molecule 1 suffers from a smaller intermolecular excitonic coupling effect induced by the $\pi-\pi$ stacking interaction due to their twisted structure. Therefore, both the emission lifetime and efficiency are enhanced.

A mechanistic picture for understanding the cause of AIE effect is necessary especially from the aspects of quantum mechanics. AIE-active silole has a twisted structure and is conformationally unstable (strained) due to the repulsion of sterically crowded phenyl rings. The intramolecular vibrations and rotations (torsions) are very active in a free state. It was conjectured that these low-frequency motions constitute the major non-radiative energy dissipation pathways in the solution state. These motions are suppressed in the aggregate state, leading to recovery of the radiative electronic transitions. We employed
Shuai's method to calculate how these low-frequency motions affect the fluorescence efficiency of $\mathbf{1}^{34-36}$ To reduce the cost of the calculation, we extracted the central luminogenic part 1,1dimethyl-2,3,4,5-tetraphenylsilole (TPS) as a model (Fig. 3d). The ground state geometry $\left(\mathrm{S}_{0}\right)$ was optimized at the level of the density functional theory (DFT) in Gaussian 03 program $^{37}$ and then the time-dependent DFT method was applied to optimize the first single excited state geometry $\left(\mathrm{S}_{1}\right)$. The B3LYP functional and 6-31G(d) basis set were used. At the equilibrium geometries, the vibrational frequencies and the normal vibrational modes of $\mathrm{S}_{0}$ electronic states are calculated by analytic energy gradients, and the ones for $S_{1}$ are obtained by numerical energy gradients. Under harmonic approximation by considering the Duschinsky rotation effect, ${ }^{34}$ the reorganization energy, which is the energy required for structural adjustments from initial to final coordinate, was calculated. The map of specific normal modes contributed to the total reorganization energy from excited to ground state is shown in Fig. 3d. The high-frequency modes $\left(1200-1700 \mathrm{~cm}^{-1}\right)$ are related to the stretching vibrations of the $\mathrm{C}-\mathrm{C}$ or $\mathrm{C}=\mathrm{C}$ bonds, while the low-frequency modes $\left(<300 \mathrm{~cm}^{-1}\right)$ are assigned to the torsional or twisting motions of peripheral phenyl rings against the central silole core (Table S3†). The histogram of energy distribution reveals that the low-frequency 
modes are dominant with a large portion about $54.8 \%$ of the total reorganization energies of TPS. This result verifies the validity of our AIE mechanism: in the solution state, active low frequency intramolecular motions of the molecules contribute a lot to inducing excitons to decay in a nonradiative manner and thus greatly enhance the internal conversion rate, leading to no/ weak light emission, whereas in the aggregate state, strong restriction of intramolecular motions (RIM) makes the radiative decay dominant, thereby turning on the light emission.

\section{Circularly polarised luminescence (CPL)}

A combination of CD and PL studies has indicated the possibility of CPL activity. We investigated the CPL behavior of 1 in various formats, such as dissolved in good solvents, suspended as aggregates in poor solvents, by natural evaporation of its solution on a quartz substrate, dispersed in a polymer matrix, and grown into regular pattern from its solution in a microfluidic channel. As shown in Fig. 4, the non-smooth film obtained by natural evaporation, doped PMMA film, and well-resolved micro-pattern display strong fluorescence under UV illumination. Because our study concentrates on the samples as aggregate particles in suspension or solid thin film, an epi-illumination (reflection mode) optical system was home-built to evaluate the CPL activity through recording the differential spontaneous emission, $\Delta I(\lambda)=I_{\mathrm{L}}(\lambda)-I_{\mathrm{R}}(\lambda)$. This setup is suitable for investigating the chirality of opaque or nontransparent samples with strong light scattering and low transmittance. Fig. $4 \mathrm{~g}$ and $4 \mathrm{~h}$ reveal the dependencies of $\Delta I$ and emission dissymmetry factor $g_{\text {em }}$ on the wavelength. The sample in pure solution state was also measured for comparison. No CPL signal was detected in good solvent solution because $\mathbf{1}$ is nonluminescent and its conformation is randomized by free intramolecular rotations. Once the molecules are aggregated as suspended particles, cast thin film, dispersed domains in a polymer matrix, and a regular micropattern, all $\Delta I$ signals become negative with dominant RCP over LCP light emission in the whole monitored spectral region. This suggests that the arrangement of the luminophores in the aggregates have preferred one-handed helical structures (vide infra). All of the CPL spectra are similar in profile. However, their $g_{\text {em }}$ values show a clear difference, indicative of the distinct packing order in their respective helical assemblies. In heterogeneous suspension and neat static cast film, the $g_{\text {em }}$ values are about -0.12 and -0.08 on average in the detected spectral window of 420-620 $\mathrm{nm}$ and show less dependence on emission wavelength. The aggregate of $\mathbf{1}$ dispersed in PMMA matrix at 10 $\mathrm{wt} \%$ has improved performance with $g_{\text {em }}$ values in the range of -0.17 to -0.13 . Surprisingly, the fabricated pattern by evaporation of DCM/toluene solution of $\mathbf{1}$ in Teflon-based microfluidic channels ${ }^{38}$ displays dramatically enhanced emission dissymmetry effect, with the $g_{\text {em }}$ value reaching about -0.32 . The confined environment in microchannels may enhance the packing order of the molecules, which results in a larger emission dissymmetry. This research provides a convenient methodology demonstrating how to use small molecules (comparatively low processability to polymers) to fabricate patterns with micro to nanometer sizes for potential optoelectronic applications such as generation of CPL lasing and information processing and storage. It should be noted that our $g_{\text {em }}$ values are two orders of



Fig. 4 Fluorescent photographs of various solid samples of $\mathbf{1}$ and study of their CPL performances. (a-f) Fluorescence microscope images under normal laboratory lighting (left panels) and UV excitation (right panels) of 1: (a and b) natural evaporation of DCE solution, (c and d) dispersion in PMMA matrix (10 $\mathrm{wt}^{\%} \%$ ), and (e and $\mathbf{f}$ ) evaporation of DCM/toluene solution in microfluidic channels on quartz substrates. (g and h) Plots of (g) $\left(I_{\mathrm{L}}-I_{\mathrm{R}}\right)$ and (h) CPL dissymmetry factor $g_{\mathrm{em}}$ versus wavelength for $\mathbf{1}$ existing in different formats: DCM solution, DCM/hexane (v/v 1/9) mixture (suspension), neat cast film from DCE solution of $2 \mathrm{mg} \mathrm{mL}^{-1}$, dispersion in polymer matrix (10 $\mathrm{wt} \%$ in PMMA), and fabricated micropattern by evaporation of DCE solution in microfluidic channels; $g_{\mathrm{em}}=$ $2\left(I_{\mathrm{L}}-I_{\mathrm{R}}\right) /\left(I_{\mathrm{L}}+I_{\mathrm{R}}\right)$, where $I_{\mathrm{L}}$ and $I_{\mathrm{R}}$ denote the left- and right-handed emission intensity, respectively. In DCM and DCM/hexane mixture, concentration of $\mathbf{1}=2 \times 10^{-4} \mathrm{M}$, excitation wavelength $=325 \mathrm{~nm}$.

magnitude higher in general than those of commonly reported systems $\left(\sim 10^{-5}-10^{-2}\right)$. In terms of both the emission efficiency and the dissymmetry factor, this self-assembled AIE system represents the best result among organic conjugated molecules without introducing any external chiral field, e.g., chiral-nematic liquid crystalline host, to enhance the helical arrangement of luminophores. Meanwhile, it is worth noting that the CPL signals of the solid samples exhibit high stability, preserving the performances even after more than half a year storage under ambient conditions.

\section{Supramolecular assembly and structural modeling}

Direct observation of the morphological structure of the aggregates of 1 can prove the formation of helical structures. Fig. 5 

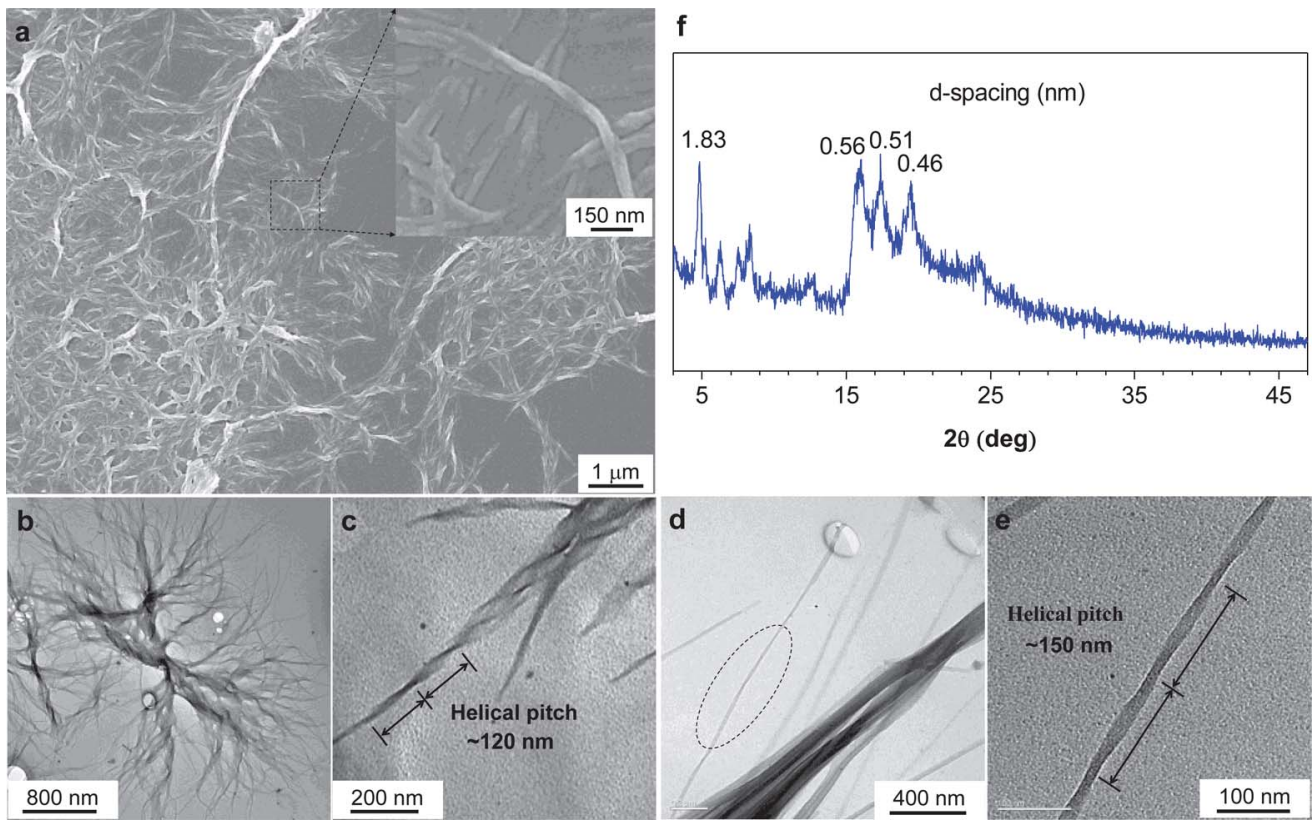

Fig. 5 Characterisation of microstructures of the assemblies of 1. (a) SEM images of the aggregates of 1 in DCM/hexane mixture (v/v 1/9). Concentration of $\mathbf{1}=2 \times 10^{-4} \mathrm{M}$. Inset: enlarged area as indicated. (b-e) TEM images of the aggregates of $\mathbf{1}$ in (b and $\left.\mathbf{c}\right) \mathrm{DCM} / \mathrm{hexane}$ mixture (v/v $\left.1 / 9\right)$, (d) from natural evaporation of DCE solution of $2 \mathrm{mg} \mathrm{mL}^{-1}$, and (e) enlarged area circled in panel d. (f) XRD diffractogram of the powder of 1 prepared from natural evaporation of its DCE solution.

shows the SEM and TEM images of the aggregates of 1 formed in $\mathrm{DCM} /$ hexane mixture with $90 \mathrm{vol} \%$ hexane fraction and by natural evaporation of its DCE solution. The molecules are assembled into predominantly right-handed helical nanoribbons with average width and helical pitch of $\sim 30$ and $\sim 120-150 \mathrm{~nm}$. The length of the helical nanoribbons can be up to micrometers.
The individual helical nanoribbons can further intertwine to form multistranded superhelical ropes with right-handed twists and lengths of several micrometers in an amorphous nature, as evidenced by electron diffraction measurement (Fig. S3†).

The self-assembly process of $\mathbf{1}$ has high instantaneity and fidelity. The fundamentals that we need to look into are how the
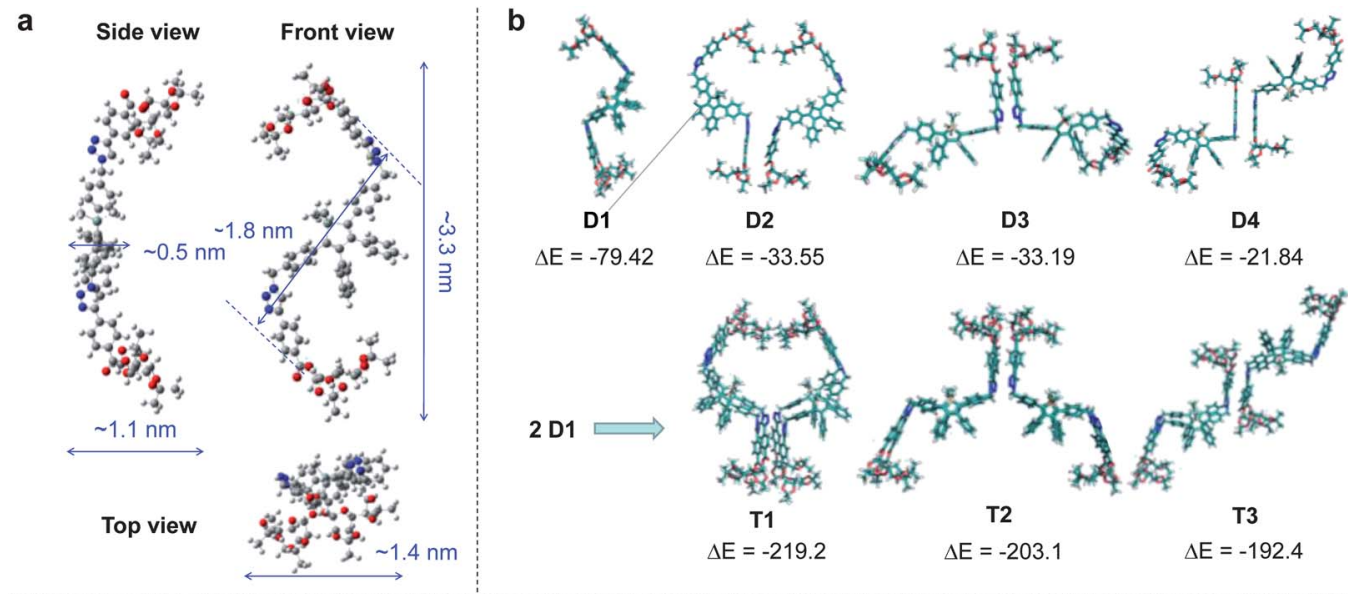

$$
\Delta \mathrm{E}=-33.55
$$
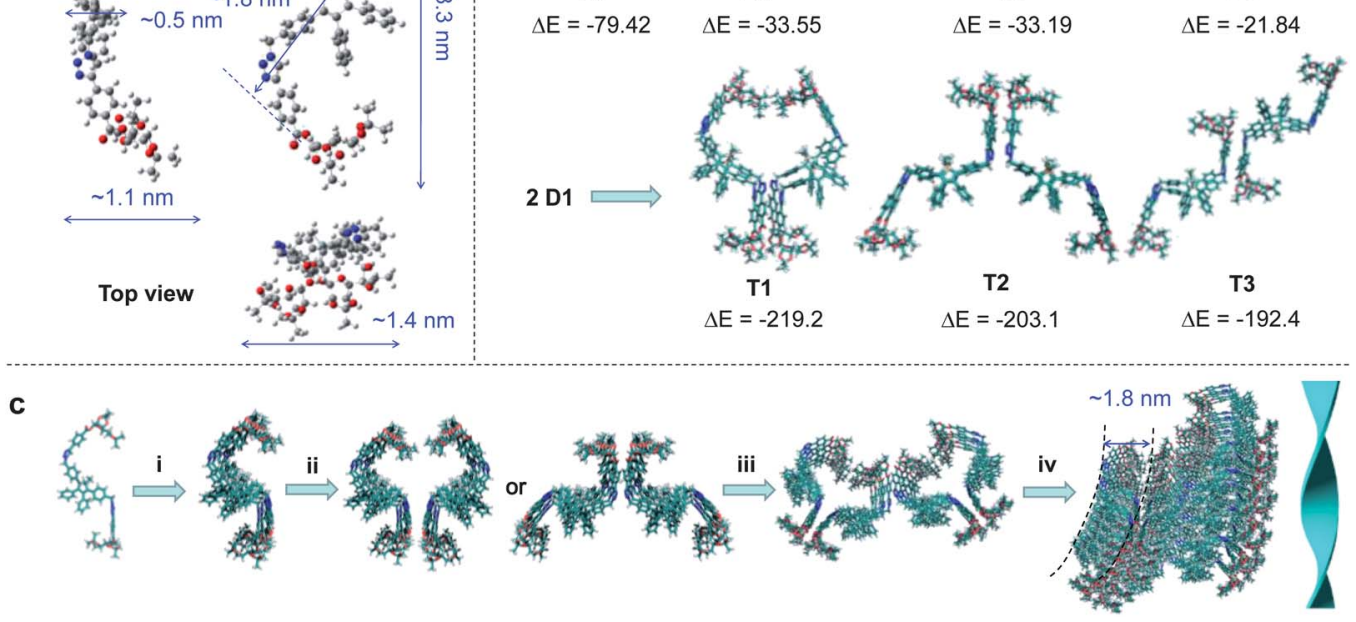

Fig. 6 Structural simulations of (a) monomer, (b) dimer and tetramer based on HF/6-31(G) level in Gaussian 03 program, and (c) proposed packing mode. (a) Different views of optimized structure of 1. (b) Energy optimization (in kJ mol ${ }^{-1}$ ) of different packing modes of dimer (D) and tetramer (T) of 1. (c) Proposed packing diagram of $\mathbf{1}$ in the assembly process: (i) single column packing, (ii) bi-column association, (iii) multi-column association, and (iv) maturation into helical nanoribbons. The representative assemblies are helical nanoribbons consisting of many associated stacked columns. 


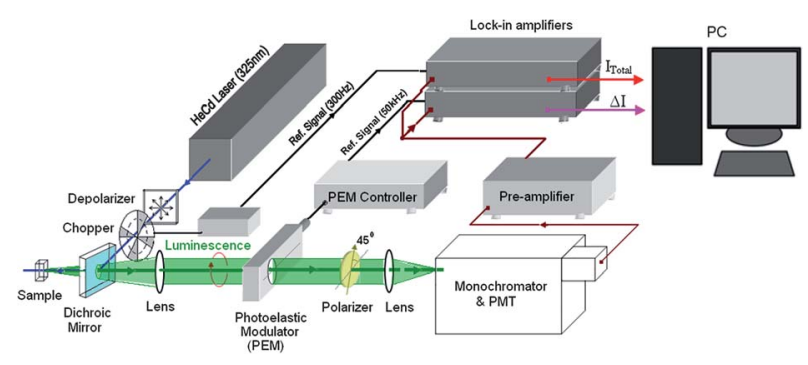

Fig. 7 Setup for the measurement of CPL.

molecules are assembled into helical ribbons and what the driving force for such process is. As seen from the molecular structure, there are no obvious functional groups showing the capacity of strong hydrogen bonding and electrostatic interactions. From the $\mathrm{CD}$ analysis of samples in the aggregate state, $\pi-$ $\pi$ stacking interaction between the silole cores to serve as the driving force can be excluded because there is no induced Cotton effect in the longer wavelength region.

Powder X-ray diffraction (XRD) technique and structural simulation were harnessed to understand the molecular packing in the helical assemblies. The powder XRD diffractogram shows the characteristic d-spacings of $\sim 0.5-0.6$ and $\sim 1.8 \mathrm{~nm}$ (Fig. $5 \mathrm{f}$ ). Correlation of the d-spacings with molecular length scales and molecular packing modes in different dimensions may offer some clues to long-range packing scenario. Based on the energy calculation at $\mathrm{HF} / 6-31(\mathrm{G})^{39}$ level in Gaussian 03 program, optimized structures of monomer 1 and their dimers and tetramers in different packing modes are shown in Fig. 6. Different views reveal its three-dimensional molecular structure and length scales (Fig. 6a). The monomer has an S-shaped conformation with a persistent length of $\sim 1.8 \mathrm{~nm}$ spanning two triazolylphenyl linkages. The calculation of dimer energies (Table S4†) with different packing modes shows that molecule 1 prefers to pile in a stacked column fashion, which has the lowest potential energy (D1, Fig. 6b). The inter-silole distance in D1 is $\sim 6.8 \AA$ (Fig. S4 $\dagger$ ), which goes beyond the working distance of $\pi-\pi$ stacking interaction between silole cores. This fact is consistent with the failed observation of induced CD signal by excitonic coupling. Two D1's are associated together to form tetramers (T1-T3) with different modes in the lateral direction in order to simulate the



Fig. 8 Schematic illustration of the fabrication process of microstrips of 1 using Teflon stamp. inter-column interaction (Fig. 6b). The result reveals that the association mode $\mathbf{T} 1$ with a close loop-like structure is dominant over the open-armed counterparts $\mathbf{T} 2$ and T3. But, their energy difference is not as large as that in the dimer packing modes, which indicates that the column-column interaction is very strong due to the collective effect and thus the energy difference in varied modes is minimized. Taking the results of structural simulation and experimental XRD data into considerations, Fig. $6 \mathrm{c}$ presents our proposed packing diagram for the assembly process. At initial stage, the molecule tends to pack at its lowest dimension to form single column structure. Accompanied with the longitudinal elongation, inter-column and multi-column association can occur in the lateral direction. Eventually, multidimensional growth leads to the maturation of the assemblies into helical nanoribbons. The nanoribbons are able to be further inter-twisted with each other to form higher-ordered superhelical ropes as evidenced in the SEM and TEM images. The d-spacings of $\sim 0.5-0.6$ and $\sim 1.8 \mathrm{~nm}$ can be properly interpreted as the intra-column molecular thickness and single column width, respectively.

What kinds of intermolecular interactions can serve as the driving force for the assembly? Two representative types of dimers D1 and D2 have been selected for close structural analysis, because they are the energy-favorable modes responsible for longitudinal and lateral growths, respectively. Careful examination manifests that there exist multiple $\mathrm{C}-\mathrm{H} \cdots \mathrm{O}(\sim 2.7 \AA)$ contacts between the paired mannose residues in both $\mathrm{D1}$ and D2. Meanwhile, $\mathrm{C}-\mathrm{H} \cdots \mathrm{N}(\sim 2.7 \AA)$ contact and weak $\pi-\pi$ stacking $(\sim 4.0 \AA)$ between two paralleled triazolylphenyl groups are involved in D1 and D2, respectively (Fig. S4 $\dagger$ ). The $\pi-\pi$ interaction in D2 will not affect the fluorescence of the silole core because its optical transitions lie in higher energy region and our utilized excitation wavelength is not able to activate them. The two pairs of interacting mannose residues in D1 accounts for its stronger interaction and thus lower potential energy compared to that of D2 with only one pair. As a result, we think that the multiple unconventional weak hydrogen bonding interactions $(\mathrm{C}-\mathrm{H} \cdots \mathrm{O}$ and $\mathrm{C}-\mathrm{H} \cdots \mathrm{N}), \pi-\pi$ stacking, and stereo-shaped complementarity between the pendants may work in a concerted manner to serve as the driving force for the assembly. Study of the detailed assembly mechanism is actively underway in our group.

\section{Conclusions}

In this work, we demonstrate how to develop molecular functional materials with high CPL performance in the condensed phase. Our designed molecule $\mathbf{1}$ has characteristics of selfassembled capacity, AICD, and unusual AIE. From solution to aggregate state, the fluorescence quantum efficiency of $\mathbf{1}$ is boosted by 136 fold $\left(\Phi_{\mathrm{F}} \sim 0.6 \%\right.$ in the solution state versus $\sim$ $81.3 \%$ in the solid state), which overcomes the serious limitations of ACQ effect encountered by conventional luminescent materials. We performed time-resolved fluorescence studies and theoretical calculation from first principles and conclude that the restriction of the low-frequency intramolecular motions is responsible for the AIE effect. In the aggregate state, the molecules tend to be assembled into right-handed helical nanoribbons and further into superhelical ropes. All the solid samples of $\mathbf{1}$ 
prefer to emit RCP light and display large CPL dissymmetry factor (absolute $g_{\text {em }}$ values $\sim 0.08-0.32$ ) and excellent spectral stability (preserved performance after more than half year storage under ambient conditions). In particular, fluorescent photopattern fabricated by Teflon-based microfluidic technique has increased the $g_{\text {em }}$ up to -0.32 due to the possibly enhanced packing order in the confined micro-environments. This technique lays foundation for using small molecules, with low processability compared to polymers, to fabricate miniaturized patterns with micro to nanometer sizes for potential optoelectronic applications. Computational simulation was carried out to understand how the molecules are organized at molecular level in the helical assemblies. Correlation of the CPL spectroscopy with its cousin $\mathrm{CD}$ provides useful database for the further development of CPL theory. As a concluding remark, this AIE-active molecule represents a class of desired molecular functional materials for generating efficient CPL in the solid state and current study shows the best result among the reported organic conjugated molecular systems in terms of emission efficiency, dissymmetry factor, and spectral stability.

\section{Experimental section}

\section{General information}

Hexane and tetrahydrofuran (THF) were distilled from sodium benzophenone ketyl immediately prior to use. Dichloromethane (DCM) was distilled over calcium hydride. Lithium wire, naphthalene, dichlorobis(triphenylphosphine)palladium(II), $\mathrm{ZnCl}_{2} \cdot \mathrm{TMEDA}$, copper(I) iodide, triphenylphosphine, and other chemicals and solvents were all purchased from Aldrich and used as received without further purification.

${ }^{1} \mathrm{H}$ and ${ }^{13} \mathrm{C}$ NMR spectra were measured on a Bruker ARX 400 NMR spectrometer using chloroform- $d$ as solvent and tetramethylsilane (TMS) as internal reference. UV absorption spectra were taken on a Milton Ray Spectronic 3000 array spectrophotometer. Crystals of $\mathbf{6}$ with quality suitable for X-ray diffraction (XRD) analysis were grown from a $1: 1 \mathrm{THF} / \mathrm{ethanol}$ mixture. Single crystal XRD intensity data were collected at $173 \mathrm{~K}$ on a Bruker-Nonius Smart Apex CCD diffractometer with graphitemonochromated Mo-K $\alpha$ radiation. The data were processed using the SAINT and SADABS routines, and the structure solution and refinement were carried out by the SHELXTL suite of X-ray programs (version 6.10). Specific optical rotations $\left([\alpha]_{\mathrm{D}}\right)$ were measured on a Perkin-Elmer 241 polarimeter at room temperature $\left(\sim 23^{\circ} \mathrm{C}\right)$ with a beam of plane-polarised light of the $\mathrm{D}$ line of a sodium lamp $(589.3 \mathrm{~nm})$ as the monochromatic source. CD spectra were recorded on a JASCO J-810 spectropolarimeter in a $1 \mathrm{~mm}$ quartz cuvette using a step resolution of $0.1 \mathrm{~nm}$, a scan speed of $100 \mathrm{~nm} \mathrm{~min}{ }^{-1}$, a sensitivity of $0.1 \mathrm{~nm}$, and a response time of $0.5 \mathrm{~s}$. PL spectra were recorded on a PerkinElmer LS 55 spectrofluorometer. Emission efficiencies of cast thin films of the molecule 1 were measured by a calibrated integrating sphere. ${ }^{40}$ High-resolution mass spectra (HRMS) were recorded on a Finnigan MAT TSQ 7000 Mass Spectrometer System operating in a MALDI-TOF mode. Thermal properties of the molecules were evaluated on TA Instruments TGA Q5000 and DSC Q1000 under nitrogen at a heating rate of $10^{\circ} \mathrm{C} \mathrm{min}^{-1}$. Morphologies and structures of the aggregates were investigated by JEOL-6300 scanning electron microscope (SEM) operating at an accelerating voltage of $15 \mathrm{kV}$ and high-resolution JEOL 2010F transmission electron microscopy (TEM).

Steady state fluorescence curves were recorded on an Edinburgh Instruments FLS920 fluorescence spectrometer. A femtosecond Ti:sapphire oscillator (150 fs pulse width and 76 $\mathrm{MHz}$ repetition rate) with the laser tuned at the second harmonic output of $358 \mathrm{~nm}$ was used as excitation laser source for the timeresolved experiment. Time-resolved PL measurements were carried out on a Hamamatsu model C4334 streak camera coupled to a spectrometer, with a time resolution of $20 \mathrm{ps.} \mathrm{PL}$ signals were collected at $500 \mathrm{~nm}$ and the laser energy level for excitation was $2 \mathrm{~mW}$. Decay in the PL intensity $(I)$ with time $(t)$ was fitted by a double-exponential function (eqn (2)) and the weighted mean lifetime $\langle\tau\rangle$ was calculated according to eqn (3):

$$
\begin{array}{r}
I=A_{1} e^{-t / \tau_{1}}+A_{2} e^{-t / \tau_{2}} \\
\langle\tau\rangle=\frac{A_{1} \tau_{1}+A_{2} \tau_{2}}{A_{1}+A_{2}}
\end{array}
$$

Circular photoluminescence spectra were measured with a home-made CPL spectroscopy system as shown in Fig. $7 .{ }^{41}$ The home-made CPL spectrometer is composed of a $325 \mathrm{~nm} \mathrm{He}-\mathrm{Cd}$ laser as the light source, a photo-elastic modulator (Hinds, PEM90, $50 \mathrm{kHz}$ ), a broad-band Glan-laser polarizer (Special Optics) oriented at 450 to the PEM optical axis, a monochromator (SPEX $500 \mathrm{M}$ ) and a photomultiplier (PMT, Hamamatsu, R928). The excitation laser of $325 \mathrm{~nm}$ in wavelength was irradiated to the sample from the same side of emission collection. A UV depolarizer was employed on the excitation light to avoid non-nature CPL. The retardation of the emitted light was controlled by the quarter-wave $50 \mathrm{kHz}$ modulator PEM-90 and detected by the photomultiplier tube after passing through the linear polarizer. The DC component of the PMT output was measured by a digital multimeter (Thurlby 1905a), while the AC component with frequency of $50 \mathrm{kHz}$ was amplified by a preamplifer (Stanford Research Systems, SR560) and analyzed by a lock-in amplifier (Stanford Research Systems, SR510). The CPL dissymmetry factor, $g_{\text {em }}=2\left(I_{\mathrm{L}}-I_{\mathrm{R}}\right) /\left(I_{\mathrm{L}}+I_{\mathrm{R}}\right)$, was evaluated from the ratio of the AC signal to the DC signal.

\section{Fabrication of micropattern}

The following flow chart depicts the fabrication process of microstrips of $\mathbf{1}$ on the quartz substrate using Teflon stamp (Fig. 8). The Teflon stamp was fabricated using the method developed by Wu's lab. ${ }^{38}$ The obtained Teflon stamp was sealed onto a clean quartz substrate using clamps. A $2 \mu \mathrm{L} 1.5 \mathrm{wt} \% \mathbf{1}$ DCM/toluene solution was added at one end of the Teflon stamp, and it filled the microchannels between the Teflon stamp and the quartz substrate with capillary force. The whole device was put in a vacuum oven to remove toluene/DCM inside the microchannels. Finally, the Teflon stamp was removed to leave the patterned microstrips on the quartz substrate.

\section{Material preparation}

Preparation of dimethylbis(phenylethynyl)silane (2). See ref. 30 for details. 
Preparation of 4-bromobenzylazide (5). Into a flask equipped with a magnetic stirrer were added 4-bromobenzyl bromide (7.5 g, $30 \mathrm{mmol})$, sodium azide $(7.8 \mathrm{~g}, 120 \mathrm{mmol})$, and $40 \mathrm{~mL}$ of DMSO. After stirred at $70{ }^{\circ} \mathrm{C}$ for $12 \mathrm{~h}$, the solution was poured into $150 \mathrm{~mL}$ of water and extracted with $\mathrm{CH}_{2} \mathrm{Cl}_{2}$. The crude product was purified by silica-gel chromatography using hexane as eluent to give a colorless viscous liquid in $96 \%$ yield $(6.12 \mathrm{~g})$. ${ }^{1} \mathrm{H} \mathrm{NMR}\left(\mathrm{CDCl}_{3}, 400 \mathrm{MHz}\right), \delta$ (TMS, ppm): 7.47 (d, $J=8.2 \mathrm{~Hz}$, $2 \mathrm{H}), 7.15(\mathrm{~d}, J=8.2 \mathrm{~Hz}, 2 \mathrm{H}), 4.26(\mathrm{~s}, 2 \mathrm{H}) .{ }^{13} \mathrm{C} \mathrm{NMR}\left(\mathrm{CDCl}_{3}\right.$, $100 \mathrm{MHz}$ ), $\delta$ (TMS, ppm): 134.3, 131.8, 129.6, 122.1, 53.9. HRMS (MALDI-TOF): $\mathrm{m} / z 210.9640\left(\mathrm{M}^{+}\right.$, calcd 210.9745).

Preparation of 1,1-dimethyl-2,5-bis[4-(azidomethyl)phenyl]-3,4diphenylsilole (6). ${ }^{31,42}$ A mixture of lithium $(0.056 \mathrm{~g}, 8 \mathrm{mmol})$ and naphthalene $(1.04 \mathrm{~g}, 8 \mathrm{mmol})$ in $8 \mathrm{~mL}$ of THF was stirred at room temperature under nitrogen for $3 \mathrm{~h}$ to form a deep dark green solution of LiNaph. A solution of $2(0.52 \mathrm{~g}, 2 \mathrm{mmol})$ in $5 \mathrm{~mL}$ of THF was then added dropwise to LiNaph solution over $4 \mathrm{~min}$ at room temperature. After stirring for $1 \mathrm{~h}$, the mixture was cooled to $0{ }^{\circ} \mathrm{C}$ and then diluted with $25 \mathrm{~mL}$ THF. A black suspension was formed upon addition of $\mathrm{ZnCl}_{2}$. TMEDA ( $2 \mathrm{~g}$, $8 \mathrm{mmol})$. After stirring for an additional hour at room temperature, a solution of $5(0.89 \mathrm{~g}, 4.2 \mathrm{mmol})$ and $\mathrm{PdCl}_{2}\left(\mathrm{PPh}_{3}\right)_{2}$ $(0.08 \mathrm{~g}, 0.1 \mathrm{mmol})$ in $25 \mathrm{~mL}$ of THF was added. The mixture was refluxed overnight. After cooled to room temperature, $100 \mathrm{~mL}$ of $1 \mathrm{M} \mathrm{HCl}$ solution was added and the mixture was extracted with DCM. The combined organic layer was washed with brine and water and then dried over magnesium sulfate. After solvent evaporation under reduced pressure, the residue was purified by a silica-gel column using hexane as eluent. The product was obtained as a yellow solid in $57 \%$ yield. ${ }^{1} \mathrm{H}$ NMR $(400 \mathrm{MHz}$, $\left.\mathrm{CDCl}_{3}\right), \delta$ (TMS, ppm): $7.06(\mathrm{~d}, J=8.1 \mathrm{~Hz}, 4 \mathrm{H}), 7.01(\mathrm{~m}, 6 \mathrm{H})$, $6.92(\mathrm{~d}, J=8.1 \mathrm{~Hz}, 4 \mathrm{H}), 6.78(\mathrm{~m}, 4 \mathrm{H}), 4.24$ (s, 4H), 0.47 (s, 6H). ${ }^{13} \mathrm{C} \mathrm{NMR}$ (100 MHz, $\mathrm{CDCl}_{3}$ ), d (TMS, ppm): 154.3, 141.3, 139.9, $138.5,132.4,129.9,129.1,127.9,127.5,126.3,54.6,-3.9$. HRMS (MALDI-TOF): $m / z$ 524.2200 $\left(\mathrm{M}^{+}\right.$, calcd 524.2145).

Preparation of 2,3:5,6-di- $O$-isopropylidene-1- $O$-(4-ethynylbenzoyl)- $\alpha$-D-mannofuranose (7). See ref. 32 for details.

Preparation of 1 . Into a $50 \mathrm{~mL}$ flask equipped with a magnetic stirrer were added $0.105 \mathrm{~g}(0.2 \mathrm{mmol})$ of 6 and $0.194 \mathrm{~g}(0.5 \mathrm{mmol})$ of 7 in $15 \mathrm{~mL}$ of water/THF/ethanol mixture (1/1/1, v/v/v). $0.5 \mathrm{~mL}$ of freshly prepared $1 \mathrm{M}$ aqueous solution of sodium ascorbate was added followed by $7.5 \mathrm{mg}(0.03 \mathrm{mmol})$ of copper(II) sulfate in $0.1 \mathrm{~mL}$ of water. The solution was stirred vigorously at $70{ }^{\circ} \mathrm{C}$ overnight. After cooled to room temperature, $50 \mathrm{~mL}$ of water was added and the reaction mixture was extracted with DCM. The combined organic layer was washed with brine and water. After solvent evaporation under reduced pressure, the residue was purified by a silica-gel column using gradient DCM/ethyl acetate $(50 / 50$ to $0 / 100, v / v)$ mixture as eluent. A yellow solid was obtained in $95.6 \%$ yield. ${ }^{1} \mathrm{H}$ NMR $\left(400 \mathrm{MHz}, \mathrm{CDCl}_{3}\right), \delta$ (TMS, ppm): 8.05 (d, $J=8.2 \mathrm{~Hz}, 4 \mathrm{H}), 7.89$ (d, $J=8.2 \mathrm{~Hz}, 4 \mathrm{H}), 7.74$ (s, $2 \mathrm{H}), 7.06(\mathrm{~d}, J=8.2 \mathrm{~Hz}, 4 \mathrm{H}), 7.01(\mathrm{~m}, 6 \mathrm{H}), 6.93(\mathrm{~d}, J=8.2 \mathrm{~Hz}$, $4 \mathrm{H}), 6.77(\mathrm{~m}, 4 \mathrm{H}), 6.38(\mathrm{~s}, 2 \mathrm{H}), 5.49(\mathrm{~s}, 4 \mathrm{H}), 4.96(\mathrm{~m}, 2 \mathrm{H}), 4.89(\mathrm{~d}$, $J=6.0 \mathrm{~Hz}, 2 \mathrm{H}), 4.45(\mathrm{~m}, 2 \mathrm{H}), 4.18-4.07(\mathrm{~m}, 6 \mathrm{H}), 1.53(\mathrm{~s}, 6 \mathrm{H})$, $1.47(\mathrm{~s}, 6 \mathrm{H}), 1.39(\mathrm{~s}, 6 \mathrm{H}), 1.38(\mathrm{~s}, 6 \mathrm{H}), 0.46(\mathrm{~s}, 6 \mathrm{H}) .{ }^{13} \mathrm{C} \mathrm{NMR}$ (100 MHz, $\mathrm{CDCl}_{3}$ ), d (TMS, ppm): 164.6, 154.8, 146.9, 141.1,
$140.5,138.3,135.4,131.4,130.4,129.8,129.4,128.8,127.7,127.5$, $126.5,125.5,120.5,113.3,109.4,101.6,85.2,82.6,79.4,72.9,66.8$, 54.1, 27.0, 25.9, 25.1, 24.6, -3.9. HRMS (MALDI-TOF): $\mathrm{m} / \mathrm{z}$ $1323.4783\left[(\mathrm{M}+\mathrm{Na})^{+}\right.$, calcd 1323.5086].

\section{Acknowledgements}

The work reported in this paper was partially supported by the National Science Foundation of China (20974028), the RPC and SRFI Grants of HKUST (RPC10SC13, RPC11SC09, and SRFI11SC03PG), the Research Grants Council of Hong Kong (604711, 603509, HKUST2/CRF/10, and N_HKUST620/11), the Innovation and Technology Commission (ITCPD/17-9) and the University Grants Committee of Hong Kong (AoE/P-03/08 and T23-713/11-1). B.Z.T. thanks the support from the Cao Guangbiao Foundation of Zhejiang University.

\section{Notes and references}

1 (a) F. S. Richardson and J. P. Riehl, Chem. Rev., 1977, 77, 773; (b) J. P. Riehl and F. S. Richardson, Chem. Rev., 1986, 86, 1.

2 (a) F. C. Spano, Z. Zhao and S. C. J. Meskers, J. Chem. Phys., 2004, 120, 10594; (b) F. C. Spano, S. C. J. Meskers, E. Hennebicq and D. Beljonne, J. Am. Chem. Soc., 2007, 129, 7044.

3 E. Gussakovsky, Circularly polarised luminescence (CPL) of proteins and protein complexes. in Reviews in Fluorescence 2008 Vol. 2008 Reviews in Fluorescence (ed Chris D. Geddes) 425-459- (Springer New York, 2010).

4 S. H. Chen, D. Katsis, A. W. Schmid, J. C. Mastrangelo, T. Tsutsui and T. N. Blanton, Nature, 1999, 397, 506.

5 A. Montali, C. Bastiaansen, P. Smith and C. Weder, Nature, 1998, 392, 261.

6 E. Peeters, M. P. T. Christiaans, R. A. J. Janssen, H. F. M. Schoo, H. P. J. M. Dekkers and E. W. Meijer, J. Am. Chem. Soc., 1997, 119, 9909.

7 (a) J. Yuasa, T. Ohno, K. Miyata, H. Tsumatori, Y. Hasegawa and T. Kawai, J. Am. Chem. Soc., 2011, 133, 9892; (b) T. Harada, Y. Nakano, M. Fujiki, M. Naito, T. Kawai and Y. Hasegawa, Inorg. Chem., 2009, 48, 11242.

8 (a) J. A. Kitchen, D. E. Barry, L. Mercs, M. Albrecht, R. D. Peacock and T. Gunnlaugsson, Angew. Chem., Int. Ed., 2012, 51, 704; (b) A. P. S. Samuel, J. L. Lunkley, G. Muller and K. N. Raymond, Eur. J. Inorg. Chem., 2010, 3343; (c) M. Seitz, K. Do, A. J. Ingram, E. G. Moore, G. Muller and K. N. Raymond, Inorg. Chem., 2009, 48, 8469

9 K. D. Oyler, F. J. Coughlin and S. Bernhard, J. Am. Chem. Soc., 2006, 129, 210.

10 S. C. J. Meskers, H. P. J. M. Dekkers, G. Rapenne and J.-P. Sauvage, Chem.-Eur. J., 2000, 6, 2129.

11 C. Schaffner-Hamann, A. von Zelewsky, A. Barbieri, F. Barigelletti, G. Muller, J. P. Riehl and A. Neels, J. Am. Chem. Soc., 2004, 126, 9339.

12 (a) T. Kaseyama, S. Furumi, X. Zhang, K. Tanaka and M. Takeuchi, Angew. Chem., Int. Ed., 2011, 50, 3684; (b) J. E. Field, G. Muller, J. P. Riehl and D. Venkataraman, J. Am. Chem. Soc., 2003, 125, 11808.

13 K. E. S. Phillips, T. J. Katz, S. Jockusch, A. J. Lovinger and N. J. Turro, J. Am. Chem. Soc., 2001, 123, 11899.

14 H. Maeda, Y. Bando, K. Shimomura, I. Yamada, M. Naito, K. Nobusawa, H. Tsumatori and T. Kawai, J. Am. Chem. Soc., 2011, 133, 9266.

15 (a) H. Tsumatori, T. Nakashima and T. Kawai, Org. Lett., 2010, 12, 2362; (b) T. Ikeda, T. Masuda, T. Hirao, J. Yuasa, H. Tsumatori, T. Kawai and T. Haino, Chem. Commun., 2012, 48, 6025.

16 K. Watanabe, T. Sakamoto, M. Taguchi, M. Fujiki and T. Nakano, Chem. Commun., 2011, 47, 10996.

17 (a) H. Hayasaka, T. Miyashita, K. Tamura and K. Akagi, Adv. Funct. Mater., 2010, 20, 1243; (b) H. Goto and K. Akagi, Angew. Chem., Int. Ed., 2005, 44, 4322.

18 S. Fukao and M. Fujiki, Macromolecules, 2009, 42, 8062. 
19 Y. Geng, A. Trajkovska, S. W. Culligan, J. J. Ou, H. M. P. Chen, D. Katsis and S. H. Chen, J. Am. Chem. Soc., 2003, 125, 14032.

20 A. Satrijo, S. C. J. Meskers and T. M. Swager, J. Am. Chem. Soc., 2006, 128, 9030.

21 J. N. Wilson, W. Steffen, T. G. McKenzie, G. Lieser, M. Oda, D. Neher and U. H. F. Bunz, J. Am. Chem. Soc., 2002, 124, 6830.

22 M. Oda, H. G. Nothofer, U. Scherf, V. Sunjić, D. Richter, W. Regenstein and D. Neher, Macromolecules, 2002, 35, 6792.

23 M. Oda, H. G. Nothofer, G. Lieser, U. Scherf, S. C. J. Meskers and D. Neher, Adv. Mater., 2000, 12, 362.

24 B. M. W. Langeveld-Voss, J. Am. Chem. Soc., 1996, 118, 4908.

25 J. B. Birks, Photophysics of Aromatic Molecules, Wiley, London, 1970.

26 J. Luo, Z. Xie, J. W. Y. Lam, L. Cheng, H. Chen, C. Qiu, H. S. Kwok, X. Zhan, Y. Liu, D. Zhu and B. Z. Tang, Chem. Commun., 2001, 1740.

27 (a) Y. Hong, J. W. Y. Lam and B. Z. Tang, Chem. Commun., 2009, 4332; (b) M. Wang, G. Zhang, D. Zhang, D. Zhu and B. Z. Tang, J. Mater. Chem., 2010, 20, 1858; (c) N.-W. Tseng, J. Liu, J. C. Y. Ng, J. W. Y. Lam, H. H. Y. Sung, I. D. Williams and B. Z. Tang, Chem. Sci., 2012, 3, 493; (d) R. Hu, J. W. Y. Lam, J. Liu, H. H. Y. Sung, I. D. Williams, Z. Yue, K. S. Wong, M. M. F. Yuen and B. Z. Tang, Polym. Chem., 2012, 3, 1481; (e) S. Chen, J. Liu, Y. Liu, H. Su, Y. Hong, C. K. W. Jim, R. T. K. Kwok, N. Zhao, W. Qin, J. W. Y. Lam, K. S. Wong and B. Z. Tang, Chem. Sci., 2012, 3, 1804; (f) J. Liu, C. Deng, N.-W. Tseng, C. Y. K. Chan, Y. Yue, J. C. Y. Ng, J. W. Y. Lam, J. Wang, Y. Hong, H. H. Y. Sung, I. D. Williams and B. Z. Tang, Chem. Sci., 2011, 2, 1850; (g) Y. Hong, L. Meng, S. Chen, C. W. T. Leung, L.-T. Da, M. Faisal, D.-A. Silva, J. Liu, J. W. Y. Lam, X. Huang and B. Z. Tang, J. Am. Chem. Soc., 2011, 134, 1680; $(h)$ H. Shi, J. Liu, J. Geng, B. Z. Tang and B. Liu, J. Am. Chem. Soc., 2012, 9569.

28 Y. Hong, J. W. Y. Lam and B. Z. Tang, Chem. Soc. Rev., 2011, 40, 5361.

29 H. C. Kolb, M. G. Finn and K. B. Sharpless, Angew. Chem., Int. Ed., 2001, 40, 2004.

30 J. Liu, Y. Zhong, J. W. Y. Lam, P. Lu, Y. Hong, Y. Yu, Y. Yue, M. Faisal, H. H. Y. Sung, I. D. Williams, K. S. Wong and B. Z. Tang, Macromolecules, 2010, 43, 4921.

31 J. Liu, J. W. Y. Lam and B. Z. Tang, J. Inorg. Organomet. Polym. Mater., 2009, 19, 249.
32 K. K. L. Cheuk, J. W. Y. Lam, B. S. Li, Y. Xie and B. Z. Tang, Macromolecules, 2007, 40, 2633.

33 N. Berova, K. Nakanishi and R. W. Woody, Circular dichroism: principles and applications (2nd Ed. 2000), Wiley-VCH.

34 Q. Peng, Y. Yi, Z. Shuai and J. Shao, J. Am. Chem. Soc., 2007, 129, 9333 .

35 Q. Peng, Y. Niu, C. Deng and Z. Shuai, Chem. Phys., 2010, 370, 215.

36 C. M. Deng, Y. Niu, Q. Peng, A. J. Qin, Z. G. Shuai and B. Z. Tang, J. Chem. Phys., 2011, 135, 014304.

37 M. J. Frisch, G. W. Trucks, H. B. Schlegel, G. E. Scuseria, M. A. Robb, J. R. Cheeseman, J. A. Montgomery, Jr., T. Vreven, K. N. Kudin, J. C. Burant, J. M. Millam, S. S. Iyengar, J. Tomasi, V. Barone, B. Mennucci, M. Cossi, G. Scalmani, N. Rega, G. A. Petersson, H. Nakatsuji, M. Hada, M. Ehara, K. Toyota, R. Fukuda, J. Hasegawa, M. Ishida, T. Nakajima, Y. Honda, O. Kitao, H. Nakai, M. Klene, X. Li, J. E. Knox, H. P. Hratchian, J. B. Cross, V. Bakken, C. Adamo, J. Jaramillo, R. Gomperts, R. E. Stratmann, O. Yazyev, A. J. Austin, R. Cammi, C. Pomelli, J. Ochterski, P. Y. Ayala, K. Morokuma, G. A. Voth, P. Salvador, J. J. Dannenberg, V. G. Zakrzewski, S. Dapprich, A. D. Daniels, M. C. Strain, O. Farkas, D. K. Malick, A. D. Rabuck, K. Raghavachari, J. B. Foresman, J. V. Ortiz, Q. Cui, A. G. Baboul, S. Clifford, J. Cioslowski, B. B. Stefanov, G. Liu, A. Liashenko, P. Piskorz, I. Komaromi, R. L. Martin, D. J. Fox, T. Keith, M. A. Al-Laham, C. Y. Peng, A. Nanayakkara, M. Challacombe, P. M. W. Gill, B. G. Johnson, W. Chen, M. W. Wong, C. Gonzalez and J. A. Pople, GAUSSIAN 03 (Revision B.01), Gaussian, Inc., Wallingford, CT, 2003.

38 K. Ren, W. Dai, J. Zhou, J. Su and H. Wu, Proc. Natl. Acad. Sci. U. S. A., 2011, 108, 8162.

39 R. McWeeny and G. Diercksen, Self-Consistent Perturbation Theory. II. Extension to Open Shells; AIP, 1968, Vol. 49.

40 Y. Kawamura, H. Sasabe and C. Adachi, Jpn. J. Appl. Phys., 2004, 43, 7729 .

41 (a) H. Tsumatori, T. Harada, J. Yuasa, Y. Hasegawa and T. Kawai, Appl. Phys. Express, 2011, 011601; (b) Y. Shindo and M. Nakagawa, Appl. Spectrosc., 1985, 39, 32.

$42 \mathrm{~K}$. Tamao, M. Uchida, T. Izumizawa, K. Furukawa and S. Yamaguchi, J. Am. Chem. Soc., 1996, 118, 11974; K. Tamao, S. Yamaguchi and M. Shiro, J. Am. Chem. Soc., 1994, 116, 11715. 